\title{
ENTREVISTA CON RALPH LEE WOODWARD JR.
}

\author{
Aharon Arguedas Zamora*
}

Palabras clave: Ralph Lee Woodward Jr., entrevista, historia, historiografía, Centroamérica.

Keywords: Ralph Lee Woodward Jr., Interview, History, Historiography, Central America.

\section{Presentación $^{1}$}

El profesor Ralph Lee Woodward Jr. ha contribuido de manera excepcional con la preparación de investigadores para el estudio de la historia centroamericana. Su indiscutible influencia se hace más que evidente en sus escritos y en importante número de estudiantes que se han dedicado a escudriñar la historia de la región.

En esta entrevista se intentará presentar las opiniones de un reconocido intelectual, maestro de generaciones. En ella se extiende sobre aspectos significativos de su vida, su formación profesional y el origen de su interés por la historia hispanoamericana. Woodward refiere, entre otras cosas, cómo se convirtió en historiador y de qué manera aumento su pasión por el desarrollo de la disciplina; además presenta las razones que lo llevaron a dedicar gran parte de su vida al estudio de Centroamérica.

1 Entrevista traducida al castellano por Katherine Jiménez Chinchilla. Correo electrónico: drakonia0790@gmail.com

Fecha de recepción: 17/02/2017 - Fecha de aceptación: 21/04/2017

* Costarricense. Doctor en Historia por Texas Christian University. Profesor e investigador de la Escuela de Historia de la Universidad Nacional. Correo electrónico: historikon@hotmail.com 


\section{Sobre Ralph Lee Woodword Jr.}

Aharon Arguedas [A.A.]: ¿Quién era Ralph Lee Woodward antes de convertirse en estudiante de historia?

Ralph Lee Woodword Jr. [RLWJ]: Yo nací en New London -Nueva Londres-, Connecticut, Estados Unidos, y mi educación primaria y secundaria la hice sobre todo en escuelas públicas de Hamden, Connecticut, cerca de New Haven. Mi padre fue profesor y asistente del decano de Divinity School en la Universidad de Yale, pero desde 1950 se convirtió en presidente de la Universidad del Centro Metodista - conocida en aquel tiempo como Central College-, en Fayette, Missouri, en donde me gradué y obtuve el grado de licenciatura en 1955.

\section{A.A.: ¿Por qué decidió estudiar historia?}

RLWJ: En mis estudios de pregrado, me especialicé en historia y ciencias políticas, con menciones en economía y español. Disfrutaba de la historia y me agradaban mis profesores, pero también por ese tiempo consideré que sería útil, para mí, ir a la Escuela de Leyes.

\section{A.A.: ¿Cómo fue esa época de su vida? ¿En qué lugares estudió?}

RLWJ: Mientras fui estudiante en Central College, el servicio militar era obligatorio en los Estados Unidos. Para poder terminar mi título universitario, me uní a las Reservas del Cuerpo de la Marina, lo cual me permitió retrasar mi servicio activo hasta después de graduarme de la universidad. Así, en 1955, se me confirió el grado de subteniente en los Cuerpos de la Marina y me desempeñé en el servicio activo en Virginia, Oklahoma, Hawái, Japón y Filipinas.

La Guerra de Corea había terminado para ese tiempo, así que mi servicio no implicó ningún combate, pero encontré útil la experiencia militar para desarrollar la autodisciplina y ordenar mi vida. Después de terminar mi servicio activo en los Cuerpos de la Marina, tuve que permanecer en las reservas hasta 1963 y, circunstancialmente, fui promovido al rango de capitán. Una vez completado mi servicio militar activo, me suscribí como estudiante de posgrado en el Departamento de Historia en la Universidad de Tulane en Nueva Orleáns, donde recibí mi Maestría en Filosofía y Letras en 1959 y mi Doctorado en Filosofía en 1962.

\section{A.A.: ¿Cuáles profesores le impresionaron e influyeron?}


RLWJ: La Facultad en Central College era conocida por su dedicación a la buena enseñanza y estrecha relación con sus estudiantes. Una influencia especial en mí fue el historiador Merrill Gaddis y mi profesor de español A. J. Cullen. Cullen me alentó a asistir a una sesión de verano en la Universidad Michoacana de San Nicolás de Hidalgo, en Morelia, México, donde desarrollé un mayor interés y amor por Latinoamérica. En la escuela de posgrado en Tulane, fui especialmente influenciado por los profesores William J. Griffith y Thomas Karnes en historia latinoamericana, pero también, en historia estadounidense, por los profesores William Hogan y Gerald Capers.

\section{A.A.: ¿Cuál fue el propósito de estudiar historia en aquel entonces? ¿Cuándo comenzó a enseñar? ¿Cuál es el objetivo hoy?}

RLWJ: Creo que la historia ha sido estudiada por mucho tiempo como un medio para entender el presente, y eso continúa siendo así hasta hoy, pero ahora hay más sofisticación metodológica y estudio especializado. Desde hace mucho tiempo existe la duda sobre si la historia es una ciencia "humana" o "social" y ese debate continúa. Por el lado de las humanidades, la historia, cuando está bien escrita y dirigida a un gran público, está muy cerca de ser "literatura" y la historiografía refleja esa división. Asumo que la historia puede ser tanto una ciencia social y humana, y creo que mi propio trabajo lo refleja. Un valor importante del estudio histórico es el desarrollo del pensamiento crítico, y eso siempre fue una meta para mí en la formación de estudiantes. Mientras más tiempo enseñaba, más incluía instrucción sobre metodología de la investigación como parte de cada clase con el fin de alentar el pensamiento crítico.

\section{A.A.: ¿Qué cambios observó en la disciplina a lo largo de su carrera?}

RLWJ: Ha habido una clara tendencia hacia la apropiación de técnicas de las ciencias sociales para los efectos del análisis histórico, tal vez menos énfasis en la biografía y la historia narrativa tradicional, que en la mayoría de los casos comprende el análisis de un fenómeno en particular.

A.A.: ¿Qué escuelas de pensamiento siguió? ¿Qué crítica podría hacer hoy a esas escuelas?

RLWJ: Yo era un poco pragmático en relación con las construcciones teóricas $\mathrm{y}$, por lo tanto, nunca adopté una escuela de pensamiento en particular. Encontré más importante, buscar objetividad y exactitud en mi escritura. Con todo, puedo decir que al principio me impresionó la desafiante respuesta 
teórica de Arnold Toynbee, en la historia de las civilizaciones, y sentí gran interés por la explicación histórica de la lucha de clases de Karl Marx. Marx me impresionó muchísimo más como historiador que por sus ideas teóricas sobre la revolución y el desarrollo futuro.

\section{A.A.: ¿Cómo fueron sus primeros años como profesor? ¿Qué consejo le daría a las nuevas generaciones historiadoras?}

RLWJ: Imitaba a los mejores profesores con los que estudié en Central College y en Tulane. Mi estilo de enseñanza era inicialmente la conferencia, pero siempre consideré de mucha importancia el estar abierto a preguntas, las cuales en muchas ocasiones condujeron a una amplia y rica discusión. No cambié mi estilo de enseñar en el curso de mi carrera, pero creo que he mejorado. En los seminarios, por supuesto, los estudiantes tuvieron un gran rol en las discusiones y presentaciones de sus ponencias. Durante mis primeros años de enseñanza, a menudo enseñé no solo varios cursos de Historia Latinoamericana, sino también cursos de historia estadounidense y de la civilización occidental, lo cual me ayudó a obtener una comprensión más amplia de la historia de mundo y a entender mejor donde encajaba Latinoamérica. Después también enseñé historia de España y Portugal.

Mi consejo para los nuevos historiadores de hoy, sería que imitaran a sus mejores profesores y que expliquen cómo sus propias investigaciones, ciertamente, se relacionan con un marco más amplio en los estudios históricos. Algo más que aprendí fue a dar conferencias sobre temas que conocía bien, dejando las lecturas asignadas para abordar y discutir los temas con los que estaba menos familiarizado.

\section{A.A.: ¿Qué historia necesitan hacer para mejorar y en qué se deben enfocar la historia?}

RLWJ: Esta es una pregunta difícil para mí, ya que he estado lejos de la enseñanza durante más de diez años, pero soy un gran creyente de las bondades de la diversidad de los enfoques y estudios de historia, de modo que no creo que haya una sola área para mejorar o enfocarse. Más bien, los jóvenes historiadores debe experimentar con su enseñanza e investigación y tratar de mejorar la relación con sus estudiantes en el salón de clases, mientras abren nuevo camino en su investigación.

\section{Sobre Centroamérica y su historia}

\section{A.A.: ¿Por qué dedicó toda su vida a estudiar Centroamérica?}


RLWJ: En realidad, no estudié exclusivamente Centroamérica. Es cierto que mis estudios de posgrado en Tulane son sobre la historia guatemalteca, aprovechando la biblioteca latinoamericana en Tulane, que es especialmente consistente en estudios sobre Guatemala, y por el consejo de mi mentor, el Dr. William J. Griffith. Mi maestría se enfocó en la organización urbana del trabajo en Guatemala (1920-54), y mi tesis doctoral en la historia del Consulado de Comercio (1793-1871). ${ }^{2}$ La mayoría de mis recientes publicaciones fueron extraídas de esas obras, aunque uno de mis primeros artículos estudió el desarrollo laboral urbano en Cuba, lugar que visité poco tiempo después de que Fidel Castro llegara al poder en $1959 .^{3}$

Después de enseñar un par de años en la Universidad de Wichita, Kansas, y en la Universidad del Suroeste de Luisiana, acepté una cátedra como asistente en la Universidad de Carolina del Norte en Chapel Hill, donde acordé enfocar mi investigación y enseñanza en el "Cono Sur", y más específicamente en Chile y Argentina. Gracias a estas becas, tuve oportunidad de pasar año y medio en Chile (1965-1966) y seis meses en Argentina (1968); dicté cursos de historia estadounidense en ambos países.

Mis investigaciones se concentraron principalmente en los consulados de Comercio de Santiago, Valparaíso y Buenos Aires, utilicé los archivos de ambos países, aunque una publicación más inmediata de ese periodo fue el libro, La isla de Robinson Crusoe: Una historia de las islas de Juan Fernández. ${ }^{4}$

En Carolina del Norte también edité una colección de lecturas sobre Positivismo en Latinoamérica, ${ }^{5}$ la cual salió poco después de mi regreso a Tulane, donde me fue ofrecida y aceptada una cátedra completa en 1970. Un factor importante en mi regreso a Nueva Orleáns fue la invitación del profesor James R. Scobie -quien estuvo investigando en el Archivo Nacional de Buenos Aires, al mismo tiempo que yo-, para escribir una historia de Centroamérica para las Series Históricas Latinoamericanas de la Universidad de Oxford Press, en lo cual él participaba en calidad de editor.

En Tulane, me enfoqué extensamente en Centroamérica, y también hice algunas investigaciones e impartí cursos en el área de las fronteras españolas, incluyendo la edición y traducción de un volumen sobre el

2 Publicado como Los privilegios de clase y desarrollo económico: El consulado de Comercio de Guatemala, 1793-1871 (Chapel Hill, EE. UU.: Universidad de Carolina del Norte Press, 1966).

3 “Trabajo urbano y comunismo: Cuba", Estudios del Caribe, 2 (1963): 363-74.

4 La isla de Robinson Crusoe: Una historia de las islas de Juan Fernández (Chapel Hill, EE. UU.: Universidad de Carolina del Norte Press), 1969.

5 Positivismo en Latinoamérica (Lexington, Mass.: D.C. Heath, 1971). 
gobernador de Luisiana Bernardo de Gálvez. ${ }^{6}$ Recientemente, también edité un interesante manuscrito de viajes del siglo XIX en México por una mujer de Nueva Orleáns, titulado Aqui y allá en México: Los viajes escritos de Mary Ashley Townsend. ${ }^{7}$

\section{A.A.: ¿Qué encontró en su primera investigación y cómo era la historia de Centroamérica en aquél entonces?}

RLWJ: Mis primeros trabajos de investigación y redacción se centraron principalmente en Guatemala, y gracias a ellos me familiaricé con la estructura social y económica en los siglos XVIII y XIX. Durante mi investigación de tesis, en Guatemala (1960-61) visité los otros Estados de Centroamérica -incluyendo Belice y Panamá-, como lo haría con frecuencia después de regresar a Tulane en 1970. Aunque mucho se había escrito sobre Centroamérica, había muy poco trabajo serio en inglés. Mi mentor Bill Griffith, a menudo enfatizó que todavía estábamos simplemente tratando de averiguar qué había sucedido en esa región, lo cual limitaba la cantidad de historia interpretativa que pudo haber sido escrita al respecto. Centroamérica era claramente un área descuidada entre especialistas latinoamericanistas en los Estados Unidos. En las décadas posteriores a la de 1960, esta insuficiencia fue subsanada en un grado sustancial, por lo que ahora hay muchos estudios norteamericanos que estudian esta región, aunque gran parte de los estudios hechos por norteamericanos, se centraron en el papel de los Estados Unidos en el istmo.

\section{A.A.: ¿Cuál fue la impresión que tuvo de la región centroamericana?}

RLWJ: Mis residencias e investigaciones en Chile y Argentina, así como en Centroamérica, me enseñaron las grandes diferencias entre los distintos Estados latinoamericanos. Estos países sudamericanos estaban entre los más desarrollados, mientras que Centroamérica, quizás con excepción de Costa Rica, estaba entre los menos. Aprendí, por tanto, a ser cuidadoso sobre hacer generalizaciones acerca de la región, empero, también encontré notables similitudes entre los países latinoamericanos, mucho más allá del lenguaje y elementos culturales en común. En suma, y por aparte, diría que las variaciones en las clases sociales y económicas entre los Estados centroamericanos han causado una gran impresión en mi trabajo histórico.

6 Homenaje a Don Bernardo de Gálvez: Patentes reales y una balada épica en honor al gobernador español de Luisiana (Baton Rouge y Nueva Orleáns, 1979).

7 Aquí y allá en México: Los viajes escritos de Mary Ashley Townsend (Tuscaloosa, EE. UU.: Universidad de Alabama Press, 2001). 


\section{A.A.: ¿Hubo algún mentor para estudiar Centroamérica?}

RLWJ: Mi mentor principal fue el profesor William J. Griffith, quien dirigió mi maestría y doctorado, y continuó ofreciéndome su asesoría y consejo en las décadas de 1960 y 1970. Otro profesor influyente de Tulane, fue Thomas L. Karnes. Karnes dejó Tulane por la Universidad del Estado de Arizona, casi al mismo tiempo que Griffith se trasladó a la Universidad de Kansas. El doctor Richard Greenleaf y yo esencialmente los reemplazamos en el Departamento de Historia de Tulane en 1970.

Griffith había construido considerablemente el Programa de Estudios Latinoamericanos en Tulane, un proceso que continuaría con Greenleaf, quien se mudó a Tulane en 1969 y sería la figura dominante en el Programa de los Estudios Latinoamericanos de Tulane durante el resto del siglo XX. Entre otros alumnos norteamericanos que influenciaron mi pensamiento acerca de la historia centroamericana, se encuentran Mario Rodríguez, Franklin Parker, Lester Langley, y Thomas Schoonover, así como también estudiantiles colegas en Tulane, incluyendo a Charles Stansifer, Guillermo Ñáñez, y John P. Bell.

\section{A.A.: ¿Fue la historiografía útil para la interpretación?}

RLWJ: Sí, por su puesto. Pasé un tiempo considerable organizando la bibliografía de la historia centroamericana porque creí que era necesario avanzar en estudios serios sobre el istmo. Esto se ve reflejado en la extensa bibliografía de Centroamérica, una nación dividida-Universidad de Oxford Press, 1976, 1985 y 1999-, así como también en volúmenes en Series bibliográficas mundiales de Clio Press que compilé en Belice (1980), Nicaragua (1983 y 1994), El Salvador (1989), y Guatemala (1992). También escribí un artículo historiográfico en la Revisión histórica de Hispanoamérica, ${ }^{8}$ la cual apareció en español como "La historiografía centroamericana moderna desde 1960". 9 Además, edité la sección centroamericana de la Guía de investigaciones para Centroamérica y el Caribe..$^{10}$

\section{A.A.: ¿Cómointerpretaustedelantesyelahora de la historia centroamericana?}

8 "The Historiography of Modern Central America since 1960", Revisión Histórica de Hispanoamérica, 67 (1987): 461-96

9 "La historiografía centroamericana moderna desde 1960", Anuario de Estudios Centroamericanos (Costa Rica) 13, n. 1 (1987): 43-65, URL: http://www.jstor.org/stable/25661913.

10 Guía de investigaciones para Centroamérica y el Caribe (Westport, Connecticut, Madison: Universidad de Wisconsin Press, 1988). 
RLWJ: Puse considerable atención en la rivalidad de liberales y conservadores en la Centroamérica del Siglo XIX, incluyendo sus raíces en el siglo XVIII y sus consecuencias en el siglo XX. Continúo considerando que las acciones de estas dos facciones de la élite ayudan a explicar el curso de la historia centroamericana en esos siglos. Esto me llevó a examinar detalladamente la evolución de estas facciones o partidos en todos los cinco países, así como también estudios biográficos de los principales participantes.

En este sentido, ciertamente, he puesto más atención a los grupos conservadores, especialmente en Guatemala y Nicaragua, y desafié las interpretaciones tradicionales liberales del siglo XIX. Estas facciones de la élite también proporcionaron pistas sobre la unión y desunión de Centroamérica a lo largo de los siglos XIX y XX.

\section{A.A.: ¿Qué se necesita desarrollar en la historia de Centroamérica?}

RLWJ: Es necesario realizar más esfuerzos para preservar los archivos públicos y privados de la región. Costa Rica lo ha logrado en un grado sustancial, pero hay mayor necesidad para tal protección en otros Estados. En lo que respecta a los temas de investigación, como ya he mencionado, estoy a favor de la visión plural y la diversificación de los estudios históricos. Creo que los estudiantes deben ser alentados a abordar nuevos temas y ser innovadores en su investigación. Sobre este particular ha sido especialmente útil, reflejando nuevas corrientes en la historia centroamericana, el Boletín de la Asociación para el Fomento de Estudios Históricos en Centroamérica, con la dirección del Dr. Christophe Belaubre, Dr. Stephen Webre y otros.

\section{A.A.: ¿Qué necesitan hacer los historiadores de la región para lograr una mejor comprensión de su historia?}

RLWJ: Los historiadores de los países de esta región son los principales escritores de la historia regional, mucho más importantes que los extranjeros como yo, que, aunque quizás hayamos hecho contribuciones a la historiografía de la región, no somos los principales historiadores de cada país. Muchos historiadores centroamericanos han recibido capacitación en universidades de Norteamérica o Europa, lo cual ha mejorado sus capacidades para hacer trabajos históricos valiosos. A su vez, ellos también han sido influyentes en quienes nos encontramos fuera de la región estudiando y escribiendo sobre el istmo.

En mi caso particular, fui especialmente influenciado por Carlos Meléndez y por otros tantos en Costa Rica, especialmente; Héctor Pérez Brignoli, Víctor Hugo Acuña, y Samuel Stone. Entre otros tantos en Guatemala, haría especial mención de Julio Castellanos Cambranes, Julio 
César Pinto Soria, Jorge Luján Muñoz, y Gustavo Palma. Ítalo López fue notablemente servicial en El Salvador, así como Rodolfo Pastor Fasquelle y Olga Joya en Honduras. Entre los varios historiadores nicaragüenses que me brindaron su ayuda destacan Jorge Eduardo Arellano y Jaime Wheelock Román, además de otros especialistas no historiadores como Xavier Zavala, Pablo Antonio Cuadra, Orlando Cuadra, Pedro Joaquín Chamorro, Daniel Ortega y Ernesto Cardenal.

A.A.: ¿Es la historia económica o del comercio la respuesta para la interpretación del pasado regional o puede entenderse mejor empleando la historia social o la microhistoria?

RLWJ: Necesitamos varios enfoques. Para mí, la historia socioeconómica ha sido más importante, pero eso no niega la gran utilidad de otros enfoques.

A.A.: ¿Centroamérica debería ser una nación unida como lo implicó en su libro?

RLWJ: Personalmente, pienso que los pueblos de la región se beneficiarían de la unión, pero eso no significa que pasará. Las ventajas en servicios sociales y comercio serían beneficios obvios, y algo de eso ya ha ocurrido. Ciertamente, Centroamérica podría desempeñar un papel más influyente en Latinoamérica si estuviera unida, pero los obstáculos y resistencias de cada país constituyen un pesado fardo.

A.A.: ¿Desde su perspectiva, fueron los cambios en el tipo de análisis histórico los que han favorecido una mejor interpretación de las décadas de 1950 a 2010 en Centroamérica?

RLWJ: Sí lo creo, en la mayor parte. La historia, en la segunda mitad del siglo $\mathrm{XX}$, implementó metodologías cada vez más rigorosas en sus estudios tanto de la región como de los distintos Estados.

\section{Sobre influencia y legados}

A.A.: Después de todos estos años, ¿cuál considera que sea el mayor legado de las universidades de los Estados Unidos a la historiografia centroamericana?

RLWJ: Creo que las universidades norteamericanas contribuyeron sustancialmente al desarrollo de una investigación y escritura más profesional de la historia centroamericana. 
Hicieron esto de diferentes modos. En primer lugar, los programas latinoamericanos de las universidades de Norteamérica capacitaron a historiadores estadounidenses y centroamericano en el estudio y escritura profesional de la historia, con especial énfasis en la objetividad y precisión. En segundo lugar, estudiantes y docentes de universidades estadounidenses llevaron a cabo investigaciones sustanciales en Centroamérica e influyeron en los académicos centroamericanos.

A.A.: ¿Los intercambios de profesores centroamericanos y norteamericanos fueron fructíferos en el campo de la interpretación y los estudios de la región?

RLWJ: Creo que sí, aunque los resultados fueron indudablemente desiguales. No solo proporcionó oportunidades a los profesores norteamericanos para que investigaran en los archivos centroamericanos y otras colecciones documentales, también proporcionó oportunidades a los académicos centroamericanos para que utilizaran algunas de las excelentes colecciones de fuentes como las de las universidades de Texas, Tulane, Kansas, California y otras, incluyendo los Archivos Nacionales de los Estados Unidos y la Biblioteca del Congreso.

A.A.: De todas sus obras, ¿cuál considera que fue la de mayor contribución al estudio de la historia centroamericana? ¿Qué lo hizo concentrar sus estudios en esta región? ¿Con qué dificultades topó?

RLWJ: Probablemente mi biografía de Rafael Carrera, ${ }^{11}$ aunque mi Centroamérica, una nación dividida fue también una contribución importante, especialmente para el estudiantado norteamericano.

Mi trabajo fue interrumpido en el 2005 por el huracán Katrina, el cual destruyó mi biblioteca y mis notas de investigación, impidiéndome terminar mi trabajo sobre la historia de los consulados de comercio en el mundo español o sobre trabajos adicionales en Centroamérica.

A.A.: ¿Por qué concentró su trabajo en el comercio o en líderes importantes? ¿Considera esto como una historia positivista?

RLWJ: No pienso que me haya concentrado especialmente en comercio o líderes importantes, aunque es verdad que gran parte de mi trabajo lo involucró.

11 Rafael Carrera and the Emergence of the Republic of Guatemala, 1821-1871 (Athens, Georgia, EE. UU.: Universidad de Georgia Press, 1993); Rafael Carrera y la creación de la República de Guatemala (1821-1871), traducción de Jorge Skinner-Kleé (Antigua Guatemala, Guatemala: CIRMA, 2002; Serviprensa Biblioteca Básica de Historia de Guatemala, 2011). 
La respuesta es que simplemente mi investigación de tesis doctoral se enfocó en comerciantes de Guatemala, y luego extendí esa problemática de trabajo en otras áreas, incluyendo la historia de los consulados de comercio a través de todo el mundo español.

En cuanto a mi biografía de Rafael Carrera, que también comenzó en mi investigación de tesis doctoral y me llevó a revisar el papel de los conservadores de Guatemala -y eventualmente en otras partes de Centroamérica-, y a desafiar las historias liberales tradicionales del siglo XIX, supongo que gran parte de mi trabajo podía decirse que se encuentra en el molde positivista, aunque es claro que mi revisión de los años conservadores puede también ser considerada como un desafío a la historiografía positivista de la región.

\section{A.A.: ¿Cuál cree que sea el mayor desafio o reto para los historiadores} centroamericanos?

RLWJ: Me gustaría ver más historiadores centroamericanos que trasciendan de sus respectivas historias nacionales y evolucionaran hacia los estudios regionales de toda Centroamérica o aún incluso en contexto latinoamericano.

\section{A.A.: ¿Cómo preparar a la próxima generación de historiadores?}

RLWJ: Alentando sus capacidades de innovación y pensamiento crítico, al mismo tiempo enseñándoles las técnicas fundamentales de investigación y escritura. Creo que especialmente los historiadores jóvenes necesitan aprender a escribir con eficacia de forma que sean entendibles no solo para colegas de su disciplina, sino también para el público en general.

\section{A.A.: ¿Cuál es el legado de Ralph Lee Woodward Jr. para la historiografía centroamericana?}

RLWJ: Dejaré que eso lo determinen otros. Quizás el legado más grande esté en todos los estudiantes a los que les impartí lecciones a lo largo de los años, incluyendo entre ellos a muchos centroamericanos como Aarón Arguedas, Rodolfo Pastor Fasquelle, Oscar Peláez Almengor, Jorge Mario Salazar, Luis Guillermo Solís, Regina Wagner, Álvaro Taboada, Enrique Gordillo, y más. Especialmente notables por su trabajo en la historia centroamericana, entre los estudiantes norteamericanos que ayudé a capacitar fueron Richmond Brown, David Carey, Paul Dosal, Kenneth Finney, Michael Fry, Virginia Garrard-Burnett, Timothy Hawkins, Heather Judge Abdelnur, Wade Kir, Sonya Lipsett, Rachel May, David McCreery, Blake Pattridge, Karen Racine, Peter Szok, John Way, Stephen Webre, y Gene Yeager. 